\title{
Caracterización de las bibliotecas universitarias en la ciudad de Medellín, Colombia
}

\section{Characterization of university libraries in Medellin, Colombia}

\author{
Luis Restrepo-Betancur \\ Universidad de Antioquia, Medellín, Colombia
}

\author{
Open Access: \\ ISSN: $0124-2121$ \\ E-ISSN: $2665-2420$ \\ ARTÍCULO RESULTADO DE \\ INVESTIGACIÓN \\ Copyright $\odot$ \\ By Educación y Humanismo \\ Editor: \\ Dhayana Fernández Matos \\ Universidad Simón Bolívar \\ Correspondencia: \\ Luis Restrepo \\ frbstatistical@yahoo.es
}

Recibido: 24-04-2020

Aceptado: 03-07-2020

En línea desde: 06-08-2020

DOI:

10.17081/eduhum.22.39.3697 


\section{Introducción}

Las bibliotecas deben propender por el desarrollo creativo de los individuos que las visitan, al igual que debe estar a tono con las nuevas metodologías de aprendizaje (Caridad et al., 2018), adaptando los nuevos desarrollos tecnológicos al servicio de los usuarios, tales como avances en internet, donde la persona pueda acceder de manera rápida y precisa a lo que anhela consultar, para lo cual debe brindar equipos computacionales que respondan de manera adecuada a la información que se requiere encontrar. De igual forma, deben contar con bases de datos sólidas, las cuales puedan servir para adelantar investigación científica de punta (Mehray Davis, 2015).

Los nuevos currículos deben contemplar, dentro de su contenido temático, un espacio de capacitación para los estudiantes, que brinde la oportunidad de adquirir conocimiento sobre la búsqueda de información bibliotecaria (Fuente, 2017). Los docentes deben incentivar a los estudiantes para que consulten plataformas que les permitan capacitarse de manera significativa mediante el uso de bibliotecas virtuales (Varela, 2013). Infortunadamente, muchas personas no pueden acceder a la información especializada que se requiere, porque no conocen de manera exacta como ingresar a la misma, y es ahí donde el personal vinculado a la biblioteca debe estar presto a responder a las inquietudes de cada individuo, a fin de que este puede contar con la información de manera oportuna y actualizada (Carrera et al., 2017).

Las bibliotecas deben estar adaptadas a las necesidades de los investigadores con relación a contar con revistas científicas de alto impacto, al igual que libros pertinentes a los requerimientos del usuario; de igual manera, deben tener servicios interbibliotecarios que les permitan acceder a información especializada, por medio de bases de datos sólidas que sirvan de apoyo para la elaboración de artículos científicos (González, 2018; Codina \& İñigo, 2015).

Una investigación llevada a cabo en España, donde se evaluaron los diferentes servicios con que cuentan los investigadores adscritos a las diferentes universidades miembros de la Red de Bibliotecas Universitarias Españolas (REBIUN), permitió destacar los siguientes aspectos: el $76 \%$ de las universidades cuenta con bibliotecas que brindan espacios de trabajo acordes con las necesidades de los investigadores; el $100 \%$ posee bases de datos especializadas, con un $95 \%$ de información bibliográfica de impacto científico; un $90 \%$ cuenta con acceso abierto; el $76 \%$ de las bibliotecas cuenta con asesoría permanente a los investigadores donde les sugieren medios para publicar; el $86 \%$ de las bibliotecas brinda servicio relacionado con derechos de autor (Rey, Camón y Pacheco, 2018).

Una de las mayores dificultades de las bibliotecas es poder tener acceso a revistas especializadas pues estas en su mayoría tienen altos costos, lo que repercute en la calidad 
de las investigaciones, por no contar con recursos económicos para acceder a ellas, y es ahí donde una buena biblioteca debe facilitar el ingreso a estas revistas ojournals. Adicionalmente, debe contar con servicios de pesquisa constante de temas de interés para los grupos de investigación por medio de acceso abierto a la información científica (Ávila, 2016).

En los servicios que prestan las bibliotecas, el bibliotecólogo juega un papel vital, caracterizándose, entre otros aspectos, por seleccionar la información pertinente para el progreso científico, y por ende de la sociedad; sirve de canal entre los investigadores y los centros de información científica, en relación con la búsqueda de datos de alto impacto; de igual manera, sirve en el diseño de bases de datos estructuradas al servicio de la docencia y la investigación. El bibliotecólogo debe ser el puente que canalice la asesoría pertinente a los diferentes usuarios que la requieran, autenticando la información que se deriva del servicio prestado (Guerra, 2019; Garciarena y Conforti, 2011).

La biblioteca pública cumple, en la actualidad, con tres roles: en primera instancia, canaliza todo lo relacionado con la cultura escrita y de información que se da de manera destacada en una sociedad; de igual forma, presenta una labor funcional, la cual está correlacionada de manera directa con las necesidades que presenta la sociedad; finalmente, brinda servicios bibliotecológicos en cada área del conocimiento, los cuales deben estar al servicio de la población objeto del servicio (Álvarez y Montoya, 2015).

Además, la biblioteca debe estar al servicio de la docencia, donde debe existir una comunicación entre los docentes y los bibliotecólogos para poder brindar mejor servicio y se puedan cumplir los objetivos de investigación de cada curso (Gallo, 2015; Marzal, Díaz y Calzada, 2012). Las bibliotecas virtuales presentan un gran impacto por la facilidad que brindan para acceder a la información. Dentro de las características destacables, deben presentar un atractivo visual y a la vez deben ser funcionales (Buerkett, 2014).

El objetivo general de la presente investigación, fue evaluar los servicios que prestan las bibliotecas universitarias en la ciudad de Medellín, contrastándolos por sexo y tipo de universidad.

\section{Método}

Se llevó a cabo un estudio descriptivo, comparativo, exploratorio de tipo multidimensional, donde las preguntas fueron de tipo cerrado en escala dicótoma (de acuerdo, en desacuerdo) y multimodal (bueno, regular y malo).

Para llevar a cabo el proceso investigativo se diseñó una encuesta de tipo estructurado de acuerdo con los objetivos planteados, teniendo en cuenta variables de tipo cualitativo asociado a patrones probabilísticos, multinomial y binomial. La encuesta se sometió a 
juicio de expertos con el objetivo de verificar la pertinencia de las preguntas y de la metodología propuesta.

La técnica de muestreo empleada fue aleatoria de proporciones en forma doblemente estratificada, por género y tipo de universidad. Se utilizó un nivel de confiabilidad del 95\% y un error máximo permisible de 3.0\%, donde los parámetros estimados $\mathrm{P}$ y $\mathrm{Q}$ adoptaron el valor del $50 \%$, debido a que no se poseen estudios anteriores referentes a la opinión sobre las bibliotecas universitarias en la ciudad de Medellín. La edad de los encuestados osciló entre los 18 a 24 años. El tamaño definitivo de la muestra fue de 1068 estudiantes pertenecientes a universidades públicas y privadas (534 estudiantes de universidad privada, 534 estudiantes de universidad pública, 534 hombres, 534 mujeres). La muestra se eligió en forma aleatoria condicionada, de acuerdo con el criterio de estratificación citado, utilizando una distribución uniforme.

La encuesta fue aplicada con participación voluntaria de los estudiantes. La base de datos se elaboró en una hoja electrónica, de acuerdo con el formato delineado en la encuesta y se realizó un estricto control de calidad en tres etapas del proceso investigativo, con el fin de garantizar los resultados y conclusiones generadas a la luz de la información.

Las preguntas relacionadas con la percepción general de la biblioteca fueron: ¿Le gusta visitar la biblioteca?, ¿Encuentra el horario de atención adecuado?, ¿Encuentra un ambiente idóneo en la biblioteca?, ¿Ha utilizado las salas de lectura?, ¿Ha solicitado ayuda para la búsqueda de material bibliográfico?, ¿Presta material bibliográfico de manera frecuente?, ¿Ha utilizado préstamo bibliográfico por envío a su hogar?, ¿Ha recibido asesoría en la búsqueda bibliotecaria?, ¿La biblioteca responde a sus necesidades de información?, ¿La atención del personal es adecuada?, ¿Conoce la distribución espacial de la biblioteca?, ¿Considera que la biblioteca permite desarrollar investigación?, ¿Los equipos computacionales son adecuados?, ¿Los docentes estimulan el uso de ella?, ¿Le estresa ir a la biblioteca?, ¿Sabe si existe manual de procedimientos?, ¿Se aplican instrumentos con regularidad sobre satisfacción suya?, ¿La biblioteca cuenta con impresoras?, ¿Tiene una PC exclusiva para la gestión bibliotecaria?, ¿Dispone de escáner la biblioteca?, ¿Existe al menos una PC de consulta?, ¿Considera el servicio de internet adecuado?, ¿Cuenta con material en CD, DVD o videos?, ¿Está automatizada la biblioteca?, ¿La comunidad educativa conoce los proyectos de la biblioteca?, ¿Se realiza préstamo a las aulas?, ¿Se exponen periódicamente las novedades que llegan?, ¿Realiza actividades de animación a la lectura?, ¿Visita la página Web de la biblioteca con alguna frecuencia?, ¿Han sido difundidas entre los usuarios las normas o reglamento?, ¿Acuden acompañados de sus docentes?, ¿Utiliza más su internet propio para consultas que ir a la biblioteca?, ¿Cada cuánto visita la biblioteca?, ¿Cuánto tiempo permanece en la biblioteca?.

Las preguntas relacionadas con el ambiente de la biblioteca fueron: ¿En la biblioteca 
existen distractores que afectan la concentración?, ¿Considera acorde la iluminación de la biblioteca?, ¿Considera confortables las sillas y las mesas de la biblioteca?, ¿El volumen de la música es acorde?, ¿El sistema de ventilación es el adecuado?, ¿Considera que el espacio de la biblioteca es el adecuado?, ¿Las estanterías son adecuadas para almacenar y exhibir libros?

Las preguntas relacionadas con los servicios de la biblioteca, fueron: Califique la sala de estudio grupal, ¿cómo percibe el servicio de audiovisuales?, ¿cómo califica la consulta de usted en la sala?, ¿cómo califica el préstamo de libros de colección general?, ¿cómo califica el préstamo de libros colección de reserva?, ¿cómo evalúa las charlas de formación de usuarios?, ¿cómo califica el acceso a bases de datos e internet?, ¿cómo evalúa la obtención de artículos y otros documentos que no están en la biblioteca?, ¿cómo califica las referencia electrónicas (internet y/o bases de datos)?, califique la agilidad en el préstamo de su biblioteca, califique la obtención de libros de otras bibliotecas vía préstamo interbibliotecario, califique los sistemas de señalización de la biblioteca, califique los servicios de referencia (orientación en búsqueda de información).

Para el análisis estadístico de los datos se empleó la técnica multivariada de la varianza MANOVA con base en el constructor de los factores, utilizando contraste canónico ortogonal, estableciendo la dimensionalidad de la comparación multidimensional, por medio del criterio de máxima verosimilitud, observando el mayor valor propio significativo. Adicionalmente, se utilizó el análisis factorial exploratorio con base en la matriz tetracórica (TCC); debido a la naturaleza de las variables, se empleó el estadístico alpha ordinal a fin de evaluar la consistencia interna. El análisis se complementó por medio de análisis factorial de correspondencia múltiple, complementándose con distribuciones de frecuencia de tipo univariado y bivariado mediante tablas de contingencia. Se utilizaron el paquete estadístico SAS UNIVERSITY y el paquete FACTOR 8.

\section{Consideraciones éticas}

Con base en la Resolución 008430 de 1993 del Ministerio de Salud de Colombia (1993), artículo 11, la investigación se clasificó sin riesgo mínimo y cumplió con todas las consideraciones éticas, donde todos los estudiantes universitarios indagados aceptaron la participación en el estudio de manera voluntaria y firmaron el consentimiento informado.

\section{Resultados y discusión}

\section{Generalidad de la biblioteca}

Se detectó diferencia significativa $(p<0.05)$ en la percepción de los estudiantes adscritos a la universidad pública, con respecto a la universidad privada, en los siguientes aspectos: la forma de percibir el ambiente de la biblioteca, donde tienen mejor concepto los estudiantes de universidad privada. En lo relacionado con el uso de 
las salas de lectura, se observó que los estudiantes de universidad pública la usan menos. Sin embargo, se encontró mayor nivel de asesoría en la búsqueda de información para los estudiantes que acuden a bibliotecas de universidades estatales, quienes adicionalmente afirman que la atención del personal vinculado a la biblioteca es mejor, donde la logística existente en la biblioteca permite desarrollar investigación científica. Además, los estudiantes afirmaron que las bibliotecas de universidades estatales cuentan con material en $C D$, DVD o videos mejores, y se exponen con mayor periodicidad las novedades que llegan (Tabla1).

El sistema de bibliotecas de la universidad privada responde mejor a las necesidades de sus usuarios. Según el concepto de los encuestados, poseen equipos más adecuados de informática, reconociendo en mayor porcentaje que existen manuales de procedimientos, se aplican en mayor medida instrumentos para evaluar la satisfacción de los estudiantes referido al sistema de biblioteca, se dispone de una PC (computadora personal) exclusiva para el manejo de la información que se deriva. Además, afirmaron tener un mejor servicio de acceso a internet al interior de la biblioteca. De igual manera, la comunidad de la universidad privada conoce de mejor forma los proyectos adelantados por la biblioteca, expresaron que brinda un mejor servicio de préstamo a las aulas y se han difundido entre los usuarios mucho mejor las normas o reglamento existentes (Tabla 1).

Al efectuar la comparación entre géneros, se detectó diferencia $(p<0.05)$ en favor del género femenino en los siguientes aspectos: la biblioteca responde mejor a sus necesidades de información, las mujeres reciben de mejor forma el mensaje emitido por los docentes referente al uso de la biblioteca, y además visitan más frecuentemente la página Web que posee la biblioteca (Tabla 1 ).

Tabla 1.

Análisis de frecuencias por tipo de universidad y género

\begin{tabular}{lcccc}
\hline \multicolumn{1}{c}{ Tema general relacionado con la biblioteca } & PU & PR & M & F \\
\hline ¿Le gusta visitar la biblioteca? & $80.6 \mathrm{a}$ & $84.6 \mathrm{a}$ & $78.6 \mathrm{a}$ & $83.8 \mathrm{a}$ \\
¿Encuentra el horario de atención adecuado? & $87.2 \mathrm{a}$ & $88.4 \mathrm{a}$ & $87.4 \mathrm{a}$ & $87.1 \mathrm{a}$ \\
¿Encuentra un ambiente idóneo dentro de la biblioteca? & $73.1 \mathrm{~b}$ & $86.5 \mathrm{a}$ & $71.0 \mathrm{a}$ & $77.5 \mathrm{a}$ \\
¿Ha utilizado las salas de lectura? & $48.0 \mathrm{~b}$ & $65.3 \mathrm{a}$ & $49.0 \mathrm{a}$ & $52.1 \mathrm{a}$ \\
¿Ha solicitado ayuda para la búsqueda de material bibliográfico? & $75.5 \mathrm{a}$ & $67.3 \mathrm{a}$ & $75.0 \mathrm{a}$ & $74.3 \mathrm{a}$ \\
¿Presta material bibliográfico de manera frecuente? & $49.9 \mathrm{a}$ & $48.0 \mathrm{a}$ & $51.0 \mathrm{a}$ & $48.1 \mathrm{a}$ \\
¿Ha utilizado préstamo bibliográfico por envío a su hogar? & $11.4 \mathrm{a}$ & $17.7 \mathrm{a}$ & $13.0 \mathrm{a}$ & $11.0 \mathrm{a}$ \\
¿Ha recibido asesoría en la búsqueda bibliotecaria? & $53.5 \mathrm{a}$ & $42.3 \mathrm{~b}$ & $52.8 \mathrm{a}$ & $50.0 \mathrm{a}$ \\
¿La biblioteca responde a sus necesidades de información? & $86.3 \mathrm{a}$ & $76.0 \mathrm{~b}$ & $79.3 \mathrm{~b}$ & $90.4 \mathrm{a}$ \\
¿La atención del personal el adecuado? & $92.7 \mathrm{a}$ & $73.0 \mathrm{~b}$ & $89.5 \mathrm{a}$ & $90.4 \mathrm{a}$ \\
¿Conoce la distribución espacial de la biblioteca? & $49.7 \mathrm{a}$ & $48.0 \mathrm{a}$ & $52.2 \mathrm{a}$ & $47.6 \mathrm{a}$ \\
¿Considera que la biblioteca permite desarrollar investigación? & $83.3 \mathrm{a}$ & $75.0 \mathrm{~b}$ & $78.4 \mathrm{a}$ & $84.7 \mathrm{a}$ \\
¿Los equipos computacionales son adecuados? & $52.7 \mathrm{~b}$ & $82.4 \mathrm{a}$ & $52.3 \mathrm{a}$ & $60.0 \mathrm{a}$ \\
¿Los docentes estimulan el uso de ella? & $65.5 \mathrm{a}$ & $57.7 \mathrm{a}$ & $59.5 \mathrm{~b}$ & $70.8 \mathrm{a}$ \\
¿Le estresa ir a la biblioteca? & $28.0 \mathrm{a}$ & $25.0 \mathrm{a}$ & $28.5 \mathrm{a}$ & $26.3 \mathrm{a}$ \\
¿Sabe si existe manual de procedimientos? & $13.1 \mathrm{~b}$ & $46.2 \mathrm{a}$ & $19.5 \mathrm{a}$ & $15.2 \mathrm{a}$
\end{tabular}


¿Se aplican instrumentos con regularidad sobre satisfacción suya? ¿La biblioteca cuenta con impresoras?

¿Tiene una PC exclusiva para la gestión bibliotecaria?

¿Dispone de escáner la biblioteca?

¿Existe al menos una PC de consulta?

¿Considera el servicio de internet adecuado?

¿Cuenta con material en CD, DVD o videos?

¿Está automatizada la biblioteca?

¿La comunidad educativa conoce los proyectos de la biblioteca?

¿Se realiza préstamo a las aulas?

¿Se exponen periódicamente las novedades que llegan?

¿Realiza actividades de animación a la lectura?

¿Visita la página Web de la biblioteca con alguna frecuencia?

¿Han sido difundidas entre los usuarios las normas o reglamento?

$27.4 \mathrm{~b}$

$38.4 a$

$28.6 a$

$30.7 a$

$24.0 \mathrm{~b}$

$35.3 a \quad 23.6 a$

$25.9 a$

$\begin{array}{llll}68.4 b & 84.6 a & 69.9 a & 70.8 a\end{array}$

$21.6 \mathrm{~b} \quad 40.4 \mathrm{a} \quad 27.1 \mathrm{a} \quad 21.5 \mathrm{a}$

$\begin{array}{llll}88.5 a & 84.6 a & 84.5 a & 90.3 a\end{array}$

$\begin{array}{llll}48.7 b & 73.0 a & 49.0 a & 53.8 a\end{array}$

$\begin{array}{llll}75.8 \mathrm{a} & 55.8 \mathrm{~b} & 70.0 \mathrm{a} & 76.3 \mathrm{a}\end{array}$

$\begin{array}{llll}59.2 \mathrm{a} & 61.5 \mathrm{a} & 56.2 \mathrm{a} & 62.5 \mathrm{a}\end{array}$

$\begin{array}{llll}33.7 \mathrm{~b} & 55.8 \mathrm{a} & 37.2 \mathrm{a} & 35.9 \mathrm{a}\end{array}$

$\begin{array}{llll}36.5 b & 53.9 a & 38.0 a & 40.1 a\end{array}$

¿Acuden acompañados de sus docentes?

$\begin{array}{llll}48.3 a & 38.5 b & 50.5 a & 45.0 a\end{array}$

$42.1 \mathrm{a} \quad 38.5 \mathrm{a} \quad 42.7 \mathrm{a} \quad 41.9 \mathrm{a}$

$33.0 a \quad 34.6 a \quad 29.5 b \quad 37.3 a$

$46.5 b \quad 61.5 a \quad 43.4 a \quad 42.8 a$

¿Utiliza más su internet propio para consultas que ir a la biblioteca?

$13.1 \mathrm{a} \quad 19.2 \mathrm{a} \quad 13.0 \mathrm{a} \quad 14.8 \mathrm{a}$

63 a 64 a 63 a 63 a

Fuente: elaboración propia. PU: público, PR: privada, M: sexo masculino, F: sexo femenino. Datos generados por los investigadores. Letras diferentes indican que existe diferencia estadística

$$
(p<0.05) \text {. }
$$

No se detectó diferencia estadística $(p>0.05)$ entre géneros, en lo relacionado con la frecuencia de visita y el tiempo de permanencia en la biblioteca (Figura 1).

Figura 1.

Distribución gráfica relacionada con la frecuencia de visita y el tiempo de permanencia en la biblioteca

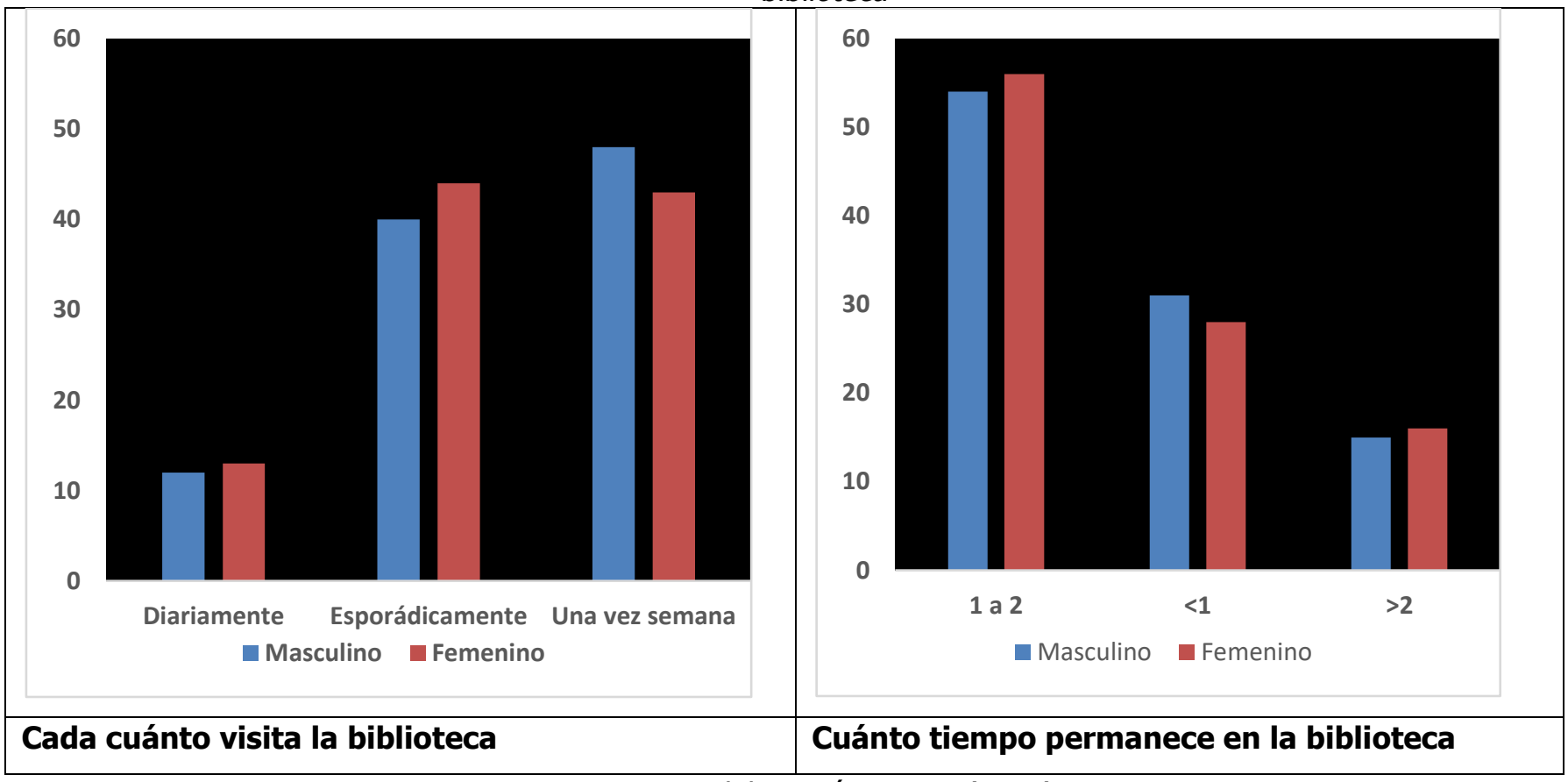

Fuente: elaboración propia (2020). 


\section{Aspectos ambientales de la biblioteca}

En lo relacionado con el tema ambiental, que comprende: iluminación, sillas, mesas, volumen de la música, sistema de ventilación, espacio de la biblioteca y estanterías existentes, se detectó diferencia estadística $(p<0.05)$ a favor de la universidad pública con respecto al confort de las sillas y mesas existentes. Sin embargo, los estudiantes consideran que existen más distractores que afectan la concentración de los estudiantes; además, consideran que el sistema de ventilación es menos adecuado. Se detectó diferencia estadística $(p<0.05)$ entre el género femenino con respecto al masculino, en lo relacionado con la sonorización musical, donde las mujeres lo consideran más acorde; además, consideran que el sistema de ventilación es más adecuado, al igual que el espacio del que disponen(Tabla 2).

Tabla 2.

Tema ambiental relacionado con la biblioteca

\begin{tabular}{lcccc}
\multicolumn{1}{c}{ Tema general relacionado con la biblioteca } & PU & PR & M & F \\
\hline ¿En la biblioteca existen distractores que afectan la concentración? & $59.0 \mathrm{a}$ & $48.0 \mathrm{~b}$ & $59.6 \mathrm{a}$ & $55.3 \mathrm{a}$ \\
¿Considera acorde la iluminación de la biblioteca? & $91.8 \mathrm{a}$ & $88.4 \mathrm{a}$ & $90.0 \mathrm{a}$ & $92.3 \mathrm{a}$ \\
¿Considera confortables las sillas y las mesas de la biblioteca? & $81.0 \mathrm{a}$ & $73.0 \mathrm{~b}$ & $78.9 \mathrm{a}$ & $80.8 \mathrm{a}$ \\
¿El volumen de la música es acorde? & $71.0 \mathrm{a}$ & $67.3 \mathrm{a}$ & $67.9 \mathrm{~b}$ & $73.7 \mathrm{a}$ \\
¿El sistema de ventilación es el adecuado? & $60.4 \mathrm{~b}$ & $71.0 \mathrm{a}$ & $58.0 \mathrm{~b}$ & $64.6 \mathrm{a}$ \\
¿Considera que el espacio de la biblioteca es el adecuado? & $63.3 \mathrm{~b}$ & $75.0 \mathrm{a}$ & $62.8 \mathrm{~b}$ & $67.0 \mathrm{a}$ \\
¿Las estanterías son adecuadas para almacenar y exhibir libros? & $75.6 \mathrm{a}$ & $66.6 \mathrm{a}$ & $73.7 \mathrm{a}$ & $76.0 \mathrm{a}$ \\
\hline
\end{tabular}

Fuente: elaboración propia (2020). Letras diferentes indican que existe diferencia estadística $(p<0.05)$.

\section{Servicios que ofrece la biblioteca}

En cuanto a la calificación de servicios que los estudiantes han utilizado, el calificativo bueno presenta diferencia estadística respecto a los demás calificativos (regular, malo), salvo en la pregunta referida a las charlas de formación de usuarios, donde no se detectó diferencia entre los ítems bueno y regular. El $87 \%$ de los estudiantes consideran que el préstamo de libros es bueno y ágil. El menor porcentaje de aceptabilidad está referido a las charlas que se brindan a los usuarios, y al préstamo de libros o revistas provenientes de otras bibliotecas. El $71 \%$ de las personas indagadas afirman que el acceso a bases de datos y al uso del internet es bueno (Tabla 3).

Tabla 3.

Servicios que ofrece la biblioteca

$\begin{array}{lccc}\text { Aspectos } & \text { Bueno } & \text { Regular } & \text { Malo } \\ \text { Salas de estudio grupal } & 70.0 \mathrm{a} & 27.0 \mathrm{~b} & 3.0 \mathrm{c} \\ \text { Servicios audiovisuales } & 62.0 \mathrm{a} & 35.0 \mathrm{~b} & 3.0 \mathrm{c} \\ \text { Consulta en sala } & 83.0 \mathrm{a} & 16.0 \mathrm{~b} & 1.0 \mathrm{c} \\ \text { Préstamo de libros colección general } & 87.0 \mathrm{a} & 12.0 \mathrm{~b} & 1.0 \mathrm{c} \\ \text { Préstamo de libros colección reserva } & 68.0 \mathrm{a} & 26.0 \mathrm{~b} & 6.0 \mathrm{c} \\ \text { Charlas de formación de usuarios } & 45.0 \mathrm{a} & 39.3 \mathrm{a} & 16.0 \mathrm{~b} \\ \text { Acceso a Bases de datos e Internet } & 71.0 \mathrm{a} & 22.0 \mathrm{~b} & 7.0 \mathrm{c} \\ \text { Obtención de artículos y otros documentos que no están en la biblioteca } & 49.0 \mathrm{a} & 40.0 \mathrm{~b} & 11.0 \mathrm{c} \\ \text { Referencia electrónica (internet y/o bases de datos) } & 71.0 \mathrm{a} & 26.0 \mathrm{~b} & 3.0 \mathrm{c}\end{array}$


Agilidad en el préstamo

Obtención de libros de otras bibliotecas vía préstamo interbibliotecario

Sistemas de señalización de la biblioteca

Servicios referencia (orientación en búsqueda de información)

$\begin{array}{lll}87.0 \mathrm{a} & 13.0 \mathrm{~b} & 0.0 \mathrm{c} \\ 56.0 \mathrm{a} & 33.0 \mathrm{~b} & 11.0 \mathrm{c} \\ 67.0 \mathrm{a} & 26.0 \mathrm{~b} & 7.0 \mathrm{c} \\ 74.0 \mathrm{a} & 24.0 \mathrm{~b} & 2.0 \mathrm{c}\end{array}$

$74.0 \mathrm{a} \quad 24.0 \mathrm{~b} \quad 2.0 \mathrm{c}$

Fuente: elaboración propia. Letras diferentes indican que existe diferencia estadística $(p<0.05)$.

El grado de satisfacción de los estudiantes universitarios está comprendido entre bueno y excelente, que supera el $60 \%$ (Figura 2).

Figura 2.

Grado de satisfacción referente a las bibliotecas

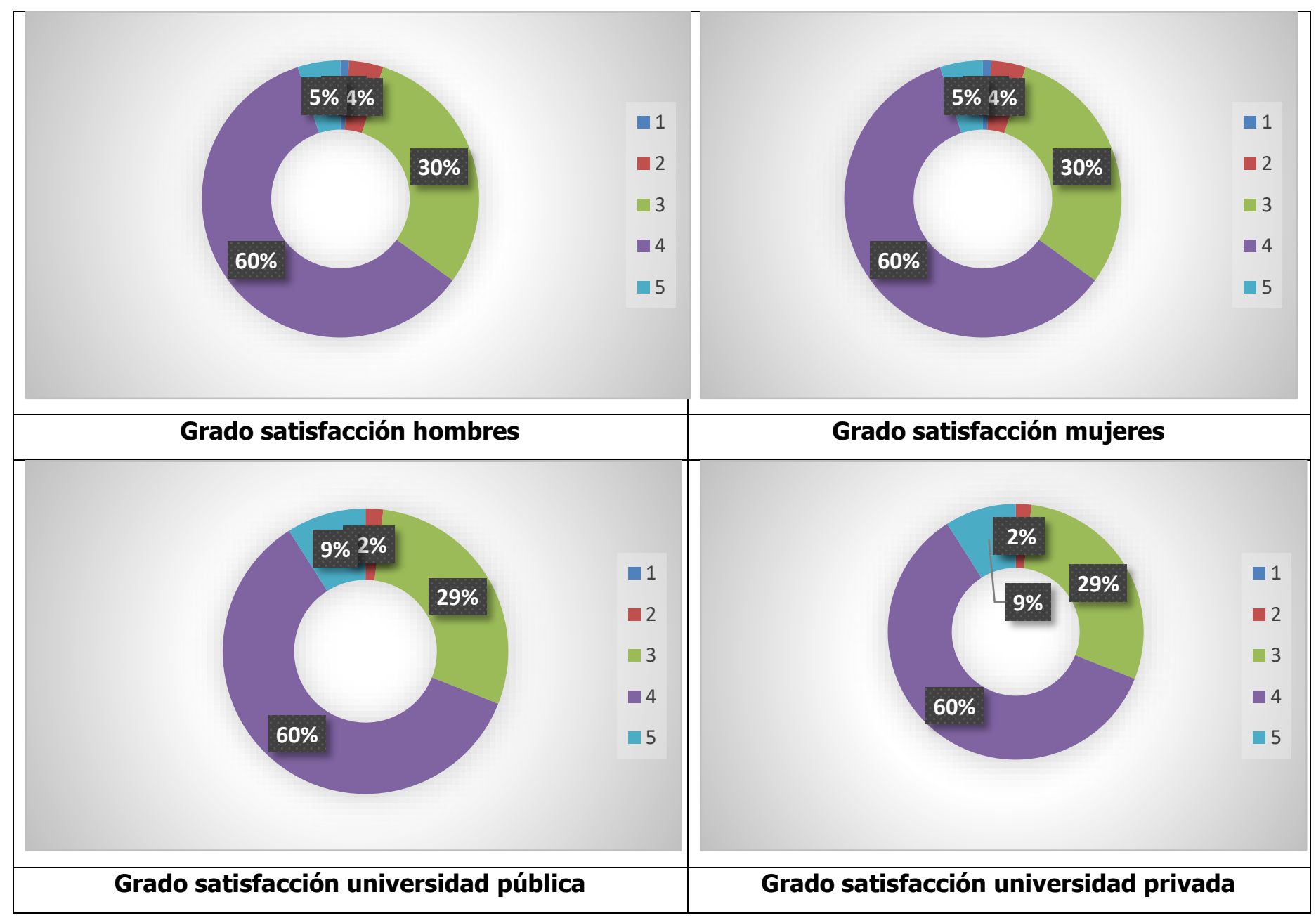

Nota: $1=$ totalmente insatisfecho, $2=$ insatisfecho, $3=$ indiferente, $4=$ satisfecho, $5=$ muy satisfecho.

\section{Análisis factorial}

Al efectuar el análisis del factor, se estableció que, en el factor uno, primaron los siguientes aspectos: ambiente idóneo de la biblioteca, equipos de sistema adecuados, internet actualizado, buen sistema de automatización de las bibliotecas, poco 
conocimiento por parte de la comunidad académica con relación a los diferentes proyectos que genera la biblioteca y las actividades de animación que efectúa. En el factor 2, pesaron las variables préstamo bibliográfico por envío al hogar o a las aulas, conocimiento espacial de la biblioteca, exposición de novedades, visita a la página Web que poseen las bibliotecas y normatividad referida por cada centro. El factor 3 se relacionó con los horarios de las bibliotecas, la asesoría que prestan a los usuarios y la atención personalizada. El factor 4 se asoció con el gusto por visitar la biblioteca, la utilización de las salas de lectura, la ayuda para buscar material bibliográfico y el préstamo general de material (Tabla 4).

Tabla 4.

Análisis del factor

\section{Tema general relacionado con la biblioteca}

¿Le gusta visitar la biblioteca?

¿Encuentra que el horario de atención es pertinente?

¿Encuentra un ambiente idóneo dentro de ella?

¿Ha utilizado las salas de lectura?

¿Ha solicitado ayuda para la búsqueda de material bibliográfico?

¿Presta material bibliográfico de manera frecuente?

¿Ha utilizado préstamo bibliográfico por envío a su hogar?

¿Ha recibido asesoría en la búsqueda bibliotecaria?

¿La biblioteca responde a sus necesidades de información?

¿La atención del personal es adecuada?

¿Conoce la distribución espacial de la biblioteca?

¿Los equipos computacionales son adecuados?

¿Considera el servicio de internet adecuado?

¿Está automatizada la biblioteca?

¿La comunidad educativa conoce los proyectos de la biblioteca?

¿Se realiza préstamo a las aulas?

¿Se exponen periódicamente las novedades que llegan?

¿Realiza actividades de animación a la lectura?

¿Visita la página Web de la biblioteca con alguna frecuencia?

¿Han sido difundidas entre los usuarios las normas o reglamento?

\section{Porcentaje explicado}

\section{F1}

F2

\section{F3}
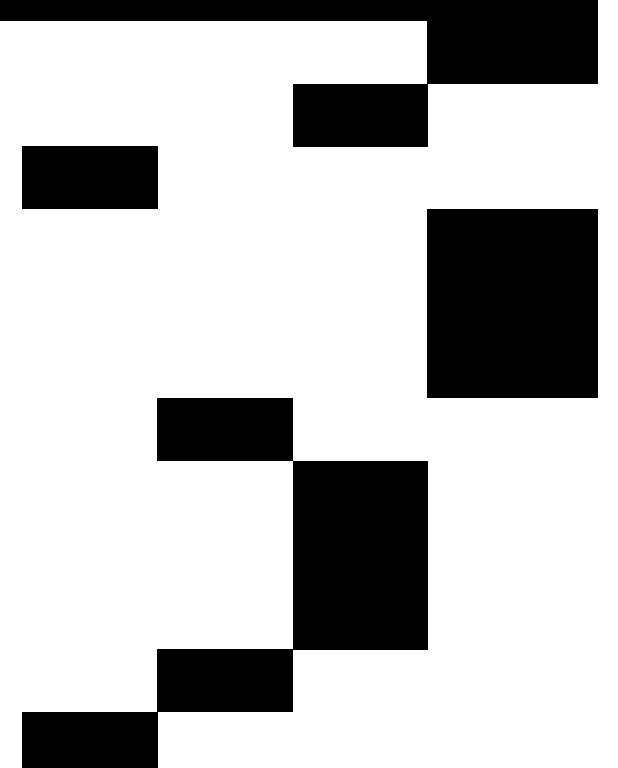
permitió determinar diferencias altamente significativas en la percepción de los diferentes aspectos referentes a la biblioteca. La diferencia se dio entre géneros y entre tipos de universidad $(p<0,0001)$, como se aprecia en la Tabla 5.

Tabla 5.

Análisis multivariado de la varianza

\begin{tabular}{lcc} 
Prueba & Género & Tipo de Universidad \\
Wilks' Lambda & $<0,0001$ & $<0,0001$ \\
Pillai's Trace & $<0,0001$ & $<0,0001$ \\
Hotelling-Lawley T & $<0,0001$ & $<0,0001$ \\
Roy'sGreatest R & $<0,0001$ & $<0,0001$ \\
\hline
\end{tabular}

Fuente: elaboración propia (2020).

\section{Discusión}

La biblioteca debe convertirse en un espacio ideal para que las personas, a temprana edad de su vida, acudan a ellas, donde la interacción con los libros sea lo más plácida y enriquecedora posible, ya que este factor permite al joven una motivación constante para acudir a futuro a ella (Lanzas, 2017; Jiménez, 2009), algo que puede incidir de forma significativa en su gusto por el aprendizaje y el conocimiento, la biblioteca debe estar al servicio de las personas marginadas y así cumplir una función social para toda la sociedad (Ceballos, 2018).

Las bibliotecas deben estar al servicio de la sociedad, procurando ir a la vanguardia en tecnologías informáticas, potencializando el intercambio interbibliotecario con el fin de brindar un mejor servicio al usuario (Romero, Gómez y Hernández, 2019). Adicionalmente, debe contar con un espacio cálido donde la persona se cautive en pro de adquirir nuevos conocimientos (Laguarde et al., 2011). El bibliotecólogo debe estar en constante capacitación, para que pueda responder a los diferentes avances que se generan en el área (Gibaja, 2013).

En un estudio llevado a cabo por Carrera et al. (2017), encontró que los docentes no incentivan a los estudiantes a acudir a la biblioteca; de manera adicional, afirman que la plataforma de la biblioteca virtual es de difícil acceso. Este es un resultado opuesto al encontrado en la presente investigación, donde un porcentaje alto de los profesores motivan a los alumnos a tener en cuenta a la biblioteca para el desarrollo de actividades de consulta. Además, los estudiantes mostraron satisfacción con las plataformas existentes en las bibliotecas de Medellín.

Una investigación llevada a cabo por Brown y Malenfant (2017), evaluó los factores existentes en las bibliotecas de una universidad de Estados Unidos y los resultados académicos de los estudiantes, donde se generó una pregunta de investigación relacionada con la misión y prioridades académicas de la universidad y la interacción entre 
la biblioteca, en aspectos tales como: colecciones con que cuenta, espacio físico y referencias existentes, entre otros aspectos y el éxito en el aprendizaje académico. Las consultas que plantean los diferentes docentes se convierten en un espacio de encuentro social, donde los bibliotecarios juegan un rol protagónico para llevar a feliz término las consultas planteadas. La biblioteca es un espacio de inclusión de la sociedad, donde se debe adaptar a cualquier tipo de persona en especial los discapacitados y los habitantes de calle (Cuadros, Valencia y Valencia, 2013; Moreno y Nairouz, 2013). En la presente investigación se encontró que a más de un $78 \%$ de los estudiantes indagados, les gusta asistir a la biblioteca, encontrando un ambiente acorde con sus expectativas, donde un alto porcentaje de alumnos han solicitado ayuda al personal de la biblioteca con el fin de encontrar los temas relacionados con las consultas propuestas, considerando que la atención brindada por el personal es muy buena. Se detectó que, a pesar de que les gusta asistir a la biblioteca, cerca del $50 \%$ de los encuestados no conocen bien los espacios físicos que posee.

Kumah (2015) efectuó un estudio en una universidad de Ghana a fin de contrastar el uso de internet con respecto a la biblioteca, donde se tenía como premisa que los alumnos emplean más internet que acudir a la biblioteca, encontrando que el empleo de la internet superó a la asistencia a la biblioteca, donde se recomendaba que esta se colocara a tono con los nuevos adelantos tecnológicos. En la presente investigación, se encontró que los estudiantes de universidades privadas emplean más las salas de lectura, frente a las personas matriculadas en universidades estatales, donde los entes privados cuentan con mejores equipos computacionales para su consulta. La mayoría de estudiantes ven en la biblioteca un espacio para propiciar la investigación. Sin embargo, utilizan más el acceso propio a internet que acudir a los servicios que presta la biblioteca.

Dentro de los aspectos negativos encontrados en esta investigación en torno a la biblioteca, se destacan: pocos estudiantes conocen los manuales de procedimientos que rigen al interior de cada centro bibliotecario, muy pocos visitan la página web con que cuenta cada biblioteca, un bajo porcentaje de docentes acuden con sus alumnos a la biblioteca, casi no cuentan con impresoras ni servicio de escáner, y la comunidad poco conoce los proyectos que adelanta la biblioteca. Los estudiantes recomiendan mejorar el servicio de internet, principalmente en las universidades estatales. Los estudiantes sugieren que debe existir mayor cantidad de charlas en lo referente a los servicios que presta cada biblioteca. Fernández et al. (2018), dicen que para atraer a las personas a la biblioteca y minimizar los aspectos negativos, se deben diseñar nueva estrategias que vinculen a la comunidad en su reestructuración.

González (2012), afirma que los servicios de referencias que las bibliotecas ofrecen a sus usuarios, tanto presenciales como virtuales, presentan bajas estadísticas, lo que pone en peligro el futuro de las bibliotecas. Es ahí donde el bibliotecario debe cumplir una función fundamental en relación con la actualización de contenidos, innovación en la búsqueda de información y atracción a los diferentes usuarios hacia los servicios que se 
brindan, siendo de gran importancia implementar un rediseño en correlación con el manejo de datos estadísticos (Malagon y Valderrama, 2016). Lo anterior implica que las bibliotecas deben adaptarse de manera constante a los nuevos desarrollos informáticos, a fin de poder potencializar las diferentes inquietudes de sus usuarios. Cerca del $90 \%$ de los ciudadanos de Estados Unidos que han sido encuestados, manifiestan su preocupación ante el posible cierre de bibliotecas, donde alrededor de un $52 \%$ de los alumnos dicen que ya no acuden tanto a la biblioteca como lo hacían antes (Rays, 2017). Los bibliotecarios deben innovar en relación a los servicios que presta la biblioteca, a fin de no desaparecer en un futuro (Anglada, 2014).

Las bibliotecas universitarias juegan un rol preponderante en la investigación que pueden adelantar tanto docentes como alumnos, facilitando la búsqueda de información para lograr los objetivos. De acuerdo con Figueredo, Figueredo y Aponte (2013), las bibliotecas:

Contribuyen, además, a la formación profesional de los estudiantes universitarios, por medio de sus servicios de información, donde se manifiesta en todo momento la vinculación de los documentos tradicionales con las tecnologías de la información y las comunicaciones, en un espacio donde confluyen el aprendizaje y el entretenimiento para un bien común. (p. 31)

En la presente investigación, se encontró que las bibliotecas universitarias privadas presentan diferencias significativas favorables en los siguientes aspectos: mejor ambiente para el estudio, mayor utilización de las salas, equipos computacionales y logísticos más adecuados. Adicionalmente, se prestan servicios en el aula y se dispone de mayor difusión de las normas y reglamento de la biblioteca. Las bibliotecas universitarias de tipo estatal, fueron mejor calificadas en lo relacionado con mayor asesoría en la búsqueda de información y mejor atención del personal; los estudiantes consideran que existe mayor posibilidad de efectuar investigación debido al material existente, además se exponen periódicamente las novedades que llegan a la biblioteca.

Al comparar la percepción sobre la biblioteca entre sexos, se encontró diferencia a favor del género femenino en lo relacionado con el mayor número de visita a la página Web; las mujeres afirman que la biblioteca responde en mayor medida a sus necesidades de información y además expresan estar más motivadas por los docentes a visitar la biblioteca.

\section{Conclusiones}

Se concluye que las bibliotecas universitarias privadas cuentan con mayor tecnología en equipos computacionales y en servicios de conexión a internet, mientras las estatales poseen mejor material bibliográfico y atención al usuario. En general, la percepción de los sexos es muy similar en lo referente al servicio que presta la biblioteca. Las mujeres tienen 
mejor concepto en el tema ambiental en lo relacionado con el volumen de la música, el espacio y el sistema de ventilación.

Las bibliotecas deben de estar en un entorno cambiante, que les permita adaptarse a los nuevos desarrollos tecnológicos, con el fin de poder brindar un servicio de calidad, al igual que deben estar indagando de manera permanente sobre los requerimientos de los usuarios.

Los currículos para profesionales en bibliotecología deben estar actualizados a las necesidades de la sociedad, a fin de que quienes egresen puedan brindar un servicio de alta calidad, que responda a las inquietudes que en el día a día se presenten.

La biblioteca debe ofrecer espacios adecuados y confortables, con el fin de que los grupos de investigación cuenten con todos los elementos técnicos y actualizados, de modo que se dinamicen y potencien las consultas requeridas, a la vez que encuentren apoyo de tipo logístico en el proceso de publicación de los adelantos que se han alcanzado.

La biblioteca virtual cada día se consolida más entre los usuarios, por la facilidad de acceso a la información, su rápida consulta y su alcance global.

Los docentes deben diseñar y asignar actividades de modo que los estudiantes necesariamente requieran acudir a la biblioteca, a fin de llevar consultas especializadas y orientadas y así incrementar una cultura bibliotecológica que les permita aumentar sus conocimientos.

Especialmente en esta época de pandemia de COVID-19, la biblioteca es fundamental en relación con los servicios virtuales que puede prestar, donde las comunidades de usuarios disponen de una amplia gama de recursos de información digital (bibliotecas de ciencias, técnicas, patrimoniales, literarias, etc.); préstamo de libros con envío a su residencia; consultas a los bibliotecarios vía internet a través de diferentes medios, como chats, correo electrónico, redes sociales (WhatsApp, Facebook, Instagram, Twitter, Linkedin, ResearchGate, entre otras); cursos formación relacionados con métodos de búsqueda de información, gestores bibliográficos, medición de impacto de las publicaciones, detección de similitud o plagio en la escritura, escritura creativa o científica, entre muchos otros servicios.

La biblioteca es fundamental para los diferentes programas a distancia que poseen algunas universidades, pero, dado que en las regiones no se dispone de suficiente material bibliográfico, estas comunidades ven limitadas sus posibilidades de acceso al material en formato físico, o se ven forzadas a desplazarse con este fin. En este sentido, la cuarentena obligatoria, con el consecuente aislamiento social al que nos vemos forzados, a la postre representa una excelente oportunidad para acelerar el necesario cambio cultural sobre la noción y la representación social de la biblioteca, motivando a los ciudadanos a conocer $y$ aprender a aprovechar el vasto mundo que nos ofrecen las 
bibliotecas virtuales. Este cambio cultural compromete igualmente a los bibliotecarios, quienes se han visto precisados a repensar la función social que cumplen, a abrir sus mentes, a aprender y a innovar para ofrecer mejores servicios, ya no solo a la persona que tiene físicamente frente a sí, sino a alguien que, literalmente aun estando al otro del mundo, en tiempo real demanda su atención.

\section{Referencias}

Álvarez, D., y Montoya, M. (2015). La biblioteca pública como sistema. Aportes a la comprensión de su condición organizacional. Revista Interamericana de Bibliotecología, 38(1), 55-64. https://revistas.udea.edu.co/index.php/RIB/article/view/21324

Anglada, L. (2014). ¿Son las bibliotecas sostenibles en un mundo de información libre, digital y en red? El profesional de la información, 23(6), 603-611. https://doi.org/10.3145/epi.2014.nov.07

Ávila, E. (2016). Aplicaciones bibliotecológicas para el acceso abierto a la información científica en el entorno digital. Revista e-Ciencias de la Información, 6(2), 1-13. http://dx.doi.org/10.15517/eci.v6i2.25340

Brown, K., \& Malenfant, K. (2017). Association of college and research libraries. Academic library impact on student learning and success: findings from assessment in action team projects. Chicago, USA: Association of College and Research Libraries. http://www.ala.org/acrl/sites/ala.org.acrl/files/content/issues/value/findings_y3.pdf

Buerkett, R. (2014). Where to start? Creating virtual library spaces. Knowledge Quest, 42(4), E23-E27. http://www.ala.org/aasl/sites/ala.org.aasl/files/content/aaslpubsandjournals/knowl edgequest/docs/KQ_MarApr_WheretoStart.pdf

Caridad, M., García, F., Martínez, S., y Morales, A. M. (2018). Bibliotecas y empoderamiento: servicios innovadores en un entorno de crisis. Revista Española de Documentación Científica, 41(2), e206. http://dx.doi.org/10.3989/redc.2018.2.1486

Carrera, O., Delgado, M., Ovando, M. C., y Contreras, E. (2017). Factores que incentivan el uso de la biblioteca virtual en los estudiantes universitarios: un estudio de caso de la Universidad de Gómez Palacio de Durango. Revista Biblios, (66), 98-111. http://dx.doi.org/10.5195/biblios.2017.333 
Ceballos, J.A. (2018). El rol de la biblioteca pública en la inclusión social del habitante de calle (homeless). Investigación Bibliotecológica, 32(76), 63-78. http://www.scielo.org.mx/pdf/ib/v32n76/2448-8321-ib-32-76-63.pdf

Codina, M., \& Íñigo, R. (2015). De la investigación al investigador. Adaptando servicios en la biblioteca Rector Gabriel Ferraté. El profesional de la información, 24(5), 648655. https://doi.org/10.3145/epi.2015.sep.13

Cuadros, R., Valencia, J., y Valencia, A. (2013). "Las bibliotecas públicas como escenarios de participación ciudadana e inclusión social". Rastros Rostros, 15(29), 73-81. https://revistas.ucc.edu.co/index.php/ra

Fernández, M., Ferran, N., Nieto, J., y Fenoll, C. (2018). La biblioteca pública vista por las personas no usuarias. El profesional de la información, 27(3). http://www.elprofesionaldelainformacion.com/contenidos/2018/may/19_esp.pdf

Figueredo, A., Figueredo, M., y Aponte, M. C. (2013). La biblioteca universitaria y el desarrollo de la educación superior. Revista Biblios, (50), 27-32. http://dx.doi.org/10.5195/biblios.2013.77

Fuente, C. (2017). Públicos vulnerables y empoderamiento digital: el reto de una sociedad e- inclusiva. El Profesional de la Información, 26(1), 5-12. http://dx.doi.org/10.3145/epi.2017.ene.01

Gallo, J. P. (2015). La biblioteca es servicio y en ello está nuestro futuro. El Profesional de la Información, 24(2), 87-93. http://dx.doi.org/10.3145/epi.2015.mar.01

Garciarena, N., y Conforti, N. (2011). La evaluación del desempeño del bibliotecario escolar en la agenda del director de la institución educativa. Revista

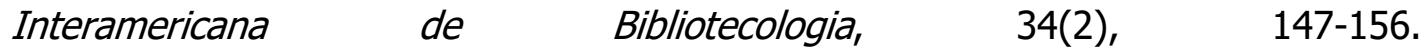
http://www.scielo.org.co/pdf/rib/v34n2/v34n2a3.pdf

Gibaja, V., (2013). El bibliotecario como profesional de la información: horizontes laborales más allá de la biblioteca. Información, Cultura y Sociedad, (29), 49-76. https://www.researchgate.net/publication/317538549_El_bibliotecario_como_profe sional_de_la_informacion_horizontes_laborales_mas_alla_de_la_biblioteca

González, N. (2012). Servicios de referencia en bibliotecas universitarias: tendencias y plan de marketing. El Profesional de la Información, 21(6), 567-576. http://dx.doi.org/10.3145/epi.2012.nov.03 
González, L. (2018). Estudios de usuarios en el diseño de servicios bibliotecarios de apoyo a la investigación: estudio de caso. Biblos, (72), 80-93. https://doi.org/10.5195/biblios.2018.427

Guerra, J. (2019). Experiencias de bibliotecólogos que laboran en bibliotecas universitarias en los procesos editoriales de revistas académicas mexicanas. Biblios, (75), 1-15. https://doi.org/10.5195/biblios.2019.467

Jiménez, Y. (2009). Aporte de la biblioteca infantil "Miriam Álvarez Brenes" y de la familia en la formación de los hábitos de lectura de la población infantil de jardines universitarios i y ii, Heredia, Costa Rica: año 2007". Bibliotecas, VXXVII (2), 133146.

Kumah, C. (2015). A comparative study of use of the library and the internet as sources of information by graduate students in the University of Ghana. Library Philosophy and Practice, (1298), 1-20. http://digitalcommons.unl.edu/libphilprac/1298

Lanzas, E. (2017). Biblioteca de aula: una propuesta didáctica para fomentar su utilización. [Trabajo de grado, Universidad Internacional de la Rioja, Facultad de Educación, Barcelona España]. Repositorio Institucional Reunir https://reunir.unir.net/bitstream/handle/123456789/5650/LANZAS\%20MEDINA\%2 C\%20ELENA. pdf?sequence $=1$ \&isAllowed $=y$

Laguarde, L.M., Gauchi, V., Peluchi, O., y Fernández, O. (2011). Condiciones ambientales en el sistema de bibliotecas públicas de la municipalidad del partido de general pueyrredon (pvcia.buenos aires-argentina): estudio de las condiciones del medio ambiente y de los factores de riesgo, y su percepción por parte de los trabajadores durante el periodo 2008-2009. Revista Chilena de Terapia Ocupacional, 11(2). https://doi.org/10.5354/0717-6767.2011.17779

Malagon, R.Y., y Valderrama, O.A. (2016). El rol del bibliotecario y la construcción del conocimiento: estrategias de articulación con instructores. Revista Rutas de $\begin{array}{lllll}\text { formación prácticas } \quad y \quad \text { experiencias, } & \text { (3), }\end{array}$ https://doi.org/10.24236/24631388.n3.2016.638

Marzal, M.A., Díaz, M.J., y Calzada, J. (2012). Un modelo y un método para la transformación de la biblioteca escolar en centro de recursos de enseñanza y $\begin{array}{lll}\text { aprendizaje. } & \text { TransInformação, 24(3), 165-178. }\end{array}$ https://www.scielo.br/pdf/tinf/v24n3/a02v24n3

Mehra, B., \& Davis, R (2015). A strategic diversity manifesto for public libraries in the 21st Century. New Library World, 116 (1/2), 15-36. https://doi.org/10.1108/NLW-04- 


\section{4-0043}

Ministerio de Salud de Colombia (1993, 4 de octubre). Resolución 008430 de 1993, Normas Científicas, Técnicas y Administrativas para la Investigación en Salud. https://www.minsalud.gov.co/sites/rid/Lists/BibliotecaDigital/RIDE/DE/DIJ/RESOLU CION-8430-DE-1993.PDF

Moreno, A., y Nairouz, Y. (2013). Uso de tecnologías de información y comunicación por personas con discapacidad en Bibliotecas Mayores de Bogotá. Revista Facultad de Medicina,

https://revistas.unal.edu.co/index.php/revfacmed/article/view/39644/47261

Raysh, T. (2017). Future of academic libraries: Opportunies and Challenges. Scholarly Research Journal for Humanity Science \& English Language, 4(24). https://doi.org/10.21922/srjhsel.v4i24.10322

Rey, C., Camón, E., y Pacheco, F. (2018). El soporte a la investigación en las bibliotecas Universitarias españolas. Anales de Documentación, 21(1). https://doi.org/10.6018/analesdoc.21.1.295841

Romero, E., Gómez, J.A., y Hernández, M. (2019). La función social de las bibliotecas públicas: la perspectiva profesional. Revista Ibero-Americana de Ciência da Informação, 12(2), 341-360. https://doi.org/10.26512/rici.v12.n2.2019.20642

Varela, C. (2013). El maestro bibliotecario como agente propulsor de la biblioteca escolar. Ibersid, (7), 103-116. https://core.ac.uk/download/pdf/290488781.pdf

\section{Agradecimentos}

A la Universidad de Antioquia, por facilitarme la labor de investigar, y a Nova, por su acompañamiento continuo.

\section{Conflicto de interés}

El autor declara que no existe conflicto de interés. 